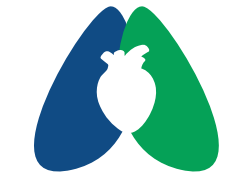

ASSOBRAFIR C I Ê N C I A

\title{
Dispositivos de Pressão Positiva Oscilatória em pacientes com Fibrose Cística um estudo de equivalência
}

\author{
Positive Oscillatory Pressure Devices in Patients with Cystic Fibrosis \\ an equivalence study
}

Francielly Dorvina Medeiros Ribeiro do Carmo'; Cristiane Cenachi Coelho'; Renata Cristina Venturato Januário'; Evanirso da Silva Aquino ${ }^{1 *}$ (1)

\section{Resumo}

Introdução: Aparelhos que auxiliam na remoção de secreção pulmonar em pneumopatas são frequentemente utilizados, entretanto há necessidade de estudar as similaridades. Objetivo: Avaliar a equivalência da aplicação de dois dispositivos de remoção das secreções de vias aéreas em pacientes com fibrose cística. Métodos: Trata-se de um estudo do tipo cruzado aleatorizado com washout, sobre a utilização dos recursos de pressão positivas oscilatórias, Shaker ${ }^{\circledR}$ e Quake ${ }^{\circledR}$. Foram selecionados crianças e adolescentes com fibrose cística com idade entre cinco e 17 anos acompanhadas em um hospital público. Antes de iniciar o estudo foi realizada a distribuição aleatória das intervenções, que aconteceram em duas sessões distintas. Os dados iniciais e finais dos participantes foram coletados. Também, foi realizada a medida do peso úmido e do peso seco e verificado a preferência quanto a utilização dos dispositivos. Um intervalo de sete dias foi considerado em cada intervenção. Resultados: Participaram do estudo nove pacientes (oito meninos e uma menina). Na comparação entre os dispositivos não foram verificadas diferenças significativas na frequência cardíaca, frequência respiratória, saturação periférica de oxigênio, peso úmido e seco da secreção expectorada. Após a intervenção com o Quake ${ }^{\circledR}$ e Shaker ${ }^{\circledR}$ houve aumento na frequência respiratória $(p=0,012)$ e no peso úmido $(p=0,036)$ na comparação com a coleta basal. A percepção subjetiva de esforço aumentou após a realização do Quake® $(p=0,04)$. Na avaliação da preferência de utilização dos dispositivos, sete pacientes relataram preferir o Quake ${ }^{\circledR}$ e um paciente o Shaker ${ }^{\circledR}$. Conclusão: O dispositivo Quake ${ }^{\circledR}$ apresentou superioridade na remoção de secreções das vias aéreas em pacientes com fibrose cística. Os voluntários relataram preferência na utilização deste dispositivo. Na avaliação dos parâmetros cardiorrespiratórios não foram encontradas diferenças estatisticamente significativas entre os dois aparelhos.

Palavras-chave: Fibrose Cística; Oscilação oral de alta frequência; Shaker; Quaker.

${ }^{1}$ Hospital Infantil João Paulo II, Belo Horizonte, MG, Brasil

\section{Como citar: Carmo FDMR, Coelho CC, Januário RCV, Aquino ES. Dispositivos de Pressão Positiva Oscilatória em pacientes com Fibrose Cística um estudo de equivalência. ASSOBRAFIR Ciênc. 2020;11:e38138. https://doi. org/10.47066/2177-9333.AC.2020.0004}

Submissão em: Outubro 10, 2019 Aceito em: Outubro 02, 2020

Estudo realizado em: Unidade de Doenças Raras, Hospital Infantil João Paulo II, Fundação Hospitalar do Estado de Minas Gerais (FHEMIG), Belo Horizonte, MG, Brasil. Aprovação ética: CAAE 70450517.3.0000.5119 parecer: 2.171.717.

*Autor correspondente:

Evanirso da Silva Aquino. E-mail: evanirsoaquino@uol.com.br

\begin{abstract}
Background: Devices to removal pulmonary secretion in lung disease patients are often used, however there is a need to study their similarities. Aim: To evaluate the equivalence of the application of two devices to remove airway secretions in patients with cystic fibrosis. Methods: This is a randomized crossover study with washout, on the use of positive oscillatory pressure resources, Shaker ${ }^{\circledR}$ and Quake ${ }^{\circledR}$. Children and adolescents with cystic fibrosis aged between five and 17 years were followed up at a public hospital. Prior to the beginning of the study, the random distribution of interventions was carried out, which took place in two different sessions and in each procedure, initial and final data were collected after the use of the devices, in addition to the measurement of wet weight, dry weight of lung mucus and preference for use. An interval of seven days was considered for each intervention. Results: Nine patients participated in the study, eight boys and one girl. In the comparison between the use of the devices, no significant differences were found in heart rate, respiratory rate, peripheral oxygen saturation, wet and dry weight. After the intervention with Quake ${ }^{\circledR}$ and Shaker ${ }^{\circledR}$ there was an increase in respiratory rate $(p=0.012)$, wet weight ( $p$ $=0.036$ ) when compared to baseline collection. Regarding the subjective perception of effort, there was an increase in this variable after Quake ${ }^{\circledR}(p=0.04)$. In assessing preference for the use of the devices, seven patients preferred the Quake ${ }^{\circledR}$ and one patient the Shaker ${ }^{\circledR}$. Conclusion: The Quake ${ }^{\circledR}$ device showed superiority in removing secretions from the airways in patients with cystic fibrosis. The volunteers reported a preference for the Quake ${ }^{\circledR}$ device. In the evaluation of cardiorespiratory parameters, no statistically significant differences were found between the devices.
\end{abstract}

Keywords: Cystic Fibrosis; High frequency oral oscillation; Shaker; Quaker.

(i) Este é um artigo publicado em acesso aberto (Open Access) e distribuído sob a licença Creative Commons Attribution NonComercial ShareAlike License, que permite uso, distribuição e reprodução em qualquer meio, sem restrições desde que sem fins comerciais e que o trabalho original seja corretamente citado e de forma que não indique endosso ao trabalho feito. Adicionalmente, qualquer trabalho derivado deverá ser publicado sob a mesma licença 


\section{INTRODUÇÃO}

A fibrose cística (FC) é uma doença genética, de caráter autossômico recessivo. É causada pela mutação do gene que codifica a proteína reguladora de condução transmembrana da FC (Cystic Fibrosis Transmembrane Conductance Regulator - CTRF), localizado no braço do cromossomo sete ${ }^{1}$. A alteração ou defeito da proteína CFTR ocasiona alteração na condutância do cloreto na membrana apical da célula epitelial. No pulmão, essa alteração provoca desidratação e aumento da viscosidade das secreções².

Nos pacientes com fibrose cística, o mecanismo da depuração mucociliar encontra-se comprometido, a principal consequência é a retenção de secreções, que levam a infecção e inflamação crônica das pequenas vias aéreas causando danos irreversíveis ${ }^{3,4}$.

Os dispositivos de pressão positiva oscilatória têm como objetivo favorecer a expectoração das secreções pulmonares. Esses recursos promovem a independência dos pacientes durante a terapia de remoção das secreções, são práticos, portáteis e contribuem para melhor adesão ao tratamento ${ }^{5}$.

Com base nesses fundamentos, destacam-se dois recursos, o Shaker ${ }^{\circledR}$ e Quake ${ }^{\circledR}$. O aparelho Shaker ${ }^{\circledR}$ foi produzido no Brasil pela empresa NCS $\AA$ e o Quake ${ }^{\circledR}$ foi criado em 2006 pela Thayer Medical, nos Estados Unidos. Ambos proporcionam vibrações nas vias aéreas, auxiliando na eliminação de secreções. O Shaker ${ }^{\circledR}$ pela movimentação da esfera de aço e o Quake ${ }^{\circledR}$ por rotação da manivela ${ }^{5,6}$. Não é de nosso conhecimento estudos que compararam os dois aparelhos analisando o peso úmido e seco da secreção expectorada e as repercussões cardiorrespiratórias em pacientes com fibrose cística.

Portanto o objetivo deste estudo foi avaliar a equivalência do Quake ${ }^{\circledR}$ e Shaker ${ }^{\circledR}$ na remoção das secreções expectoradas, a preferência dos voluntários quanto a utilização dos dispositivos e a sua influência nos parâmetros cardiorrespiratórios dos pacientes com fibrose cística.

\section{MÉTODOS}

Trata-se de um estudo do tipo cruzado aleatorizado com washout, sobre a utilização de dois dispositivos de pressão positivas oscilatórias, Shaker ${ }^{\circledR}$ e Quake ${ }^{\circledR}$. O estudo foi aprovado pelo comitê de ética e pesquisa CAEE 70450517.3.0000.5119. A coleta foi realizada no período de Julho de 2017 a Janeiro de 2018. Foram selecionados crianças e adolescentes com fibrose cística com idade entre 5 e 17 anos, acompanhadas no Ambulatório de Doenças Raras do Hospital Infantil João Paulo II da Fundação Hospitalar do Estado de Minas Gerais após a assinatura do termo de consentimento pelos pais ou responsáveis e o termo de assentimento assinados pelos pacientes. Foram excluídos do estudo aqueles indivíduos que não conseguiram realizar os dispositivos propostos; além de pacientes que apresentaram quatro sinais ou mais de exacerbação aguda da doença de acordo com Bradley et al. ${ }^{7}, 2001$ como: (a) aumento da quantidade de secreção (> $20 \mathrm{~g} / \mathrm{dia}$ ), (b) aumento ou novo episódio de sangramento nasal, (c) aumento da tosse, e (d) presença de dispneia, mal-estar, fadiga, febre, perda de peso, perda do apetite, letargia, dor em região dos seios da face e mudança no aspecto da coriza nasal; presença de colonização por Burkholderia cepacia; presença de hemoptise ou que se recusaram a participar do estudo?.

A sequência de utilização dos dispositivos foi realizada por sorteio pelo programa Random $®$ gerador de números aleatórios. O dispositivo Shaker ${ }^{\circledR}$ foi identificado como 1 e o Quake ${ }^{\circledR}$ como 2 e foram utilizados de acordo com a ordem do sorteio, com um intervalo de tempo de uma semana entre eles. Todos os pacientes utilizaram os dois dispositivos em momentos diferentes. Inicialmente foram mensurados os parâmetros cardiorrespiratórios e os participantes foram orientados a tossir cinco vezes para coleta de secreção (coleta basal). Em seguida, em uma única sessão realizaram a intervenção com o dispositivo selecionado em cinco séries de 20 repetições $^{6}$ e eram orientados a expectorar no recipiente de coleta. Após a intervenção os dados finais foram mensurados. Entre a utilização dos dispositivos houve um washout de 7 dias. A ordem de realização dos procedimentos está representada na Figura 1.

Os dispositivos foram utilizados de acordo com as orientações do fabricante. O Shaker ${ }^{\circledR}$ é um dispositivo em forma de cachimbo, que possui um cone circular, e uma esfera de aço inoxidável. A expiração no bocal do aparelho provoca a subida e descida da esfera, gerando uma pressão positiva oscilante ${ }^{8}$. Durante a utilização do Shaker ${ }^{\circledR}$ o paciente foi orientado a sentar-se em uma cadeira com encosto com cotovelos apoiados, segurando o aparelho com firmeza, mantendo o bocal na posição horizontal. Em seguida realizou uma inspiração lenta e profunda com uma pausa de 2 a 3 segundos e expirou todo o ar pela boca tranquilamente. Os indivíduos foram instruídos a realizar 5 séries com 20 repetições, com 1 minuto de descanso entre as séries. Além disso, os pacientes foram orientados a tossir e expectorar a secreção a cada série ou quando sentissem vontade.

O Quake ${ }^{\circledR}$ é um dispositivo que também possui formato de cachimbo, com uma manivela giratória, que é operada manualmente, e cria oscilações. É composto por cata-ventos internos e tonel externo com cata-ventos concorrentes, que são responsáveis pela geração da pressão positiva oscilatória9 . A frequência de oscilação é controlada pela rapidez com que a manivela é operada 10. Para utilização do dispositivo os pacientes foram orientados a sentar-se em uma cadeira com encosto, com o cotovelo apoiado, segurar o dispositivo com uma mão e com a outra rodar a manivela, em seguida associar a respiração com a rotação da manivela. 


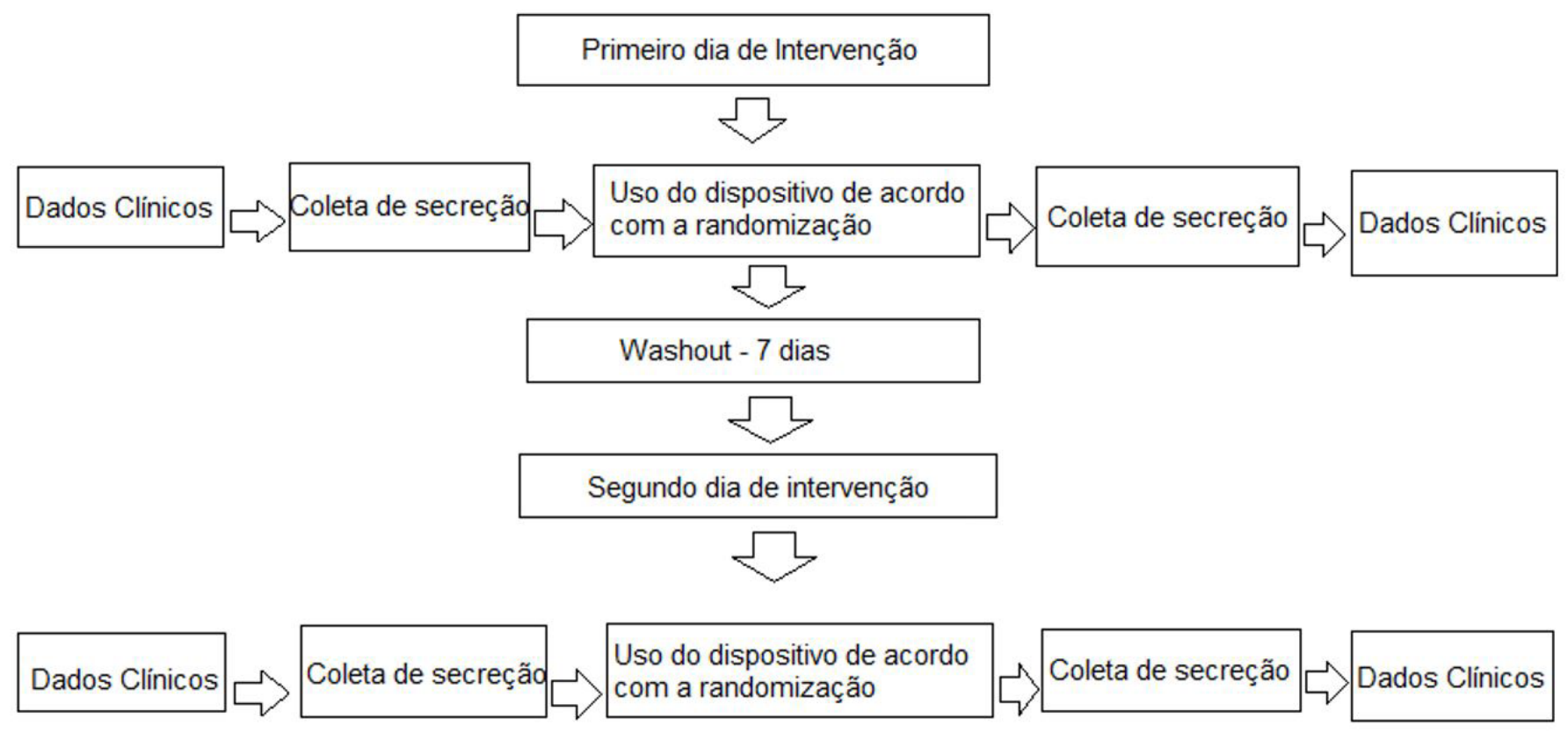

Figura 1. Desenho do estudo.

Todos os sujeitos incluídos no protocolo receberam os dispositivos, que eram novos e de uso individual.

Os dados vitais, foram avaliados antes e após a realização dos recursos e incluíram saturação periférica de oxigênio $\left(\mathrm{SpO}_{2}\right)$, frequência cardíaca $(\mathrm{Fc})$, frequência respiratória (Fr) e percepção subjetiva de esforço (Escala de Borg Modificada). A frequência cardíaca foi mensurada por um cardiofrequencímetro (POLAR ElectroOy, Model 90440, Kempele, Finlândia). A saturação periférica de oxigênio, por um oxímetro (Nonim Medical, INC Model 9500 Finger Pulse Oximeter, USA) e a Fr foi mensurada pelo avaliador, durante 60 segundos.

A secreção eliminada pelos pacientes antes e após o uso do dispositivo foi coletada em recipientes distintos, para mensurar o peso úmido e o peso seco da secreção. O peso das secreções foi avaliado logo após a sessão com cada dispositivo em uma balança analítica de precisão. Em seguida as amostras foram colocadas em uma estufa a $80^{\circ} \mathrm{C}$. Após duas horas o peso da secreção seca foi medido ${ }^{6}$. Este procedimento foi realizado no mesmo dia da coleta do material. Ao final do protocolo o paciente deveria relatar a sua preferência quanto à utilização dos dispositos através de um questionário onde a resposta seria assinalada sobre o dispositivo de escolha.

\section{ANÁLISE ESTATÍSTICA}

O tamanho da amostra foi determinado através do softwar $G$ - power versão 3.1 para duas amostras dependentes com tamanho de efeito de 3,25, poder de $80 \%$ e alfa de 0,05 , totalizando oito sujeitos.

Para análise estatística foi utilizado o propgrama SPSS versão 17.0. Na análise descritiva, o teste de normalidade
Shapiro-Wilk demonstrou distribuição assimétrica e diante disso testes não-paramétricos. Para a comparação entre os dispositivos Shaker ${ }^{\circledR}$ e Quake ${ }^{\circledR}$ e para a comparação pré e pós intervenção de cada dispositivo foi utilizado o teste de Wilcoxon e o valor de p considerado foi de 0,05. Os dados foram apresentados de forma descritiva pela mediana e quartil 50.

\section{RESULTADOS}

Participaram do estudo nove pacientes acompanhados no ambulatório de fibrose cística (oito meninos e uma menina). No decorrer do estudo houve a perda amostral de um paciente, que apresentou pneumotórax no sexto dia após a intervenção com o Shaker ${ }^{\circledR}$.

$\mathrm{Na}$ Tabela 1 estão representadas as características dos voluntários. A mutação genética e a colonização bacteriana mais frequente foram: $\Delta 508$ homozigotos em 37,5\% dos pacientes e Pseudomonas aeruginosa em 50\%, respectivamente. A doença pulmonar foi classificada de acordo com o valor do $\mathrm{VEF}_{1}$ (volume expiratório forçado de primeiro segundo). Três indivíduos apresentaram distúrbio moderado, quatro distúrbio grave e um indivíduo apresentou função pulmonar normal'.

A Tabela 2 apresenta os dados dos dispositivos Shaker ${ }^{\circledR}$ e Quake ${ }^{\circledR}$ em relação ao peso úmido e peso seco, além dos parâmetros iniciais e finais das variáveis cardiorrespiratórias. Na comparação entre a intervenção Shaker ${ }^{\circledR}$ e Quake ${ }^{\circledR}$, não foram observados valores estatisticamente significativos para as variáveis peso úmido, peso seco, $\mathrm{Fc}$, Fr, Borg e $\mathrm{SpO}_{2}$.

O Gráfico 1 apresenta o delta de variação do peso seco e peso úmido dos dispositivos Shaker ${ }^{\circledR}$ e Quake ${ }^{\circledR}$ 
Tabela 1. Características dos participantes do estudo.

\begin{tabular}{|c|c|c|c|c|c|c|c|c|c|}
\hline \multirow[b]{2}{*}{$\mathrm{N}$} & \multirow[b]{2}{*}{ Genética } & \multirow[b]{2}{*}{$\begin{array}{l}\text { Idade } \\
\text { (anos) }\end{array}$} & \multirow[b]{2}{*}{$\begin{array}{l}\text { Peso } \\
\text { (Kg) }\end{array}$} & \multirow[b]{2}{*}{$\begin{array}{l}\text { Altura } \\
(\mathrm{cm})\end{array}$} & \multirow[b]{2}{*}{$\begin{array}{c}\mathrm{IMC} \\
\left(\mathrm{kg} / \mathrm{m}^{2}\right)\end{array}$} & \multirow[b]{2}{*}{ Colonização } & \multicolumn{3}{|c|}{ Função Pulmonar } \\
\hline & & & & & & & CVF\% & VEF\% & $\begin{array}{l}\text { CVF/ } \\
\text { VEF\% }\end{array}$ \\
\hline 1 & c.579+1G>T; 508del & 14 & 48 & 1,64 & 17,8 & Pseudomonas aeruginosa & 97,5 & 96,6 & 106,7 \\
\hline 2 & 508del;c.1680-1G>A & 9 & 26 & 1,39 & 13,4 & $\begin{array}{c}\text { Stenotrophomonas } \\
\text { maltophilia }\end{array}$ & 57,9 & 47,9 & 87,3 \\
\hline 3 & c.3196C>T, 508del & 13 & 36 & 1,48 & 16,4 & Staphylococcus aureus & 62,1 & 44,3 & 75,9 \\
\hline 4 & c. $16244 \mathrm{G}>\mathrm{T} ; \mathrm{c} .1680-1 \mathrm{G}>\mathrm{A}$ & 15 & 30 & 1,39 & 15,5 & Pseudomonas aeruginosa & 29,7 & 25,6 & 92 \\
\hline 5 & 508del, 508del & 10 & 36 & 1,45 & 17,1 & Staphylococcus aureus & 63,4 & 57 & 95,2 \\
\hline 6 & c.1624G>T(homozigoto) & 14 & 29 & 1,45 & 13,7 & $\begin{array}{l}\text { Pseudomonas aeroginosa } \\
\text { mucoide }\end{array}$ & 36,9 & 29,5 & 88,2 \\
\hline 7 & 508del (homozigoto) & 13 & 28 & 1,47 & 19,0 & $\begin{array}{l}\text { Staphylococcus aureus } \\
\text { resistente a meticilina/ } \\
\text { Pseudomonas aeruginosa }\end{array}$ & 38,3 & 27,4 & 75,9 \\
\hline 8 & 508del(homozigoto) & 12 & 29 & 1,42 & 14,3 & $\begin{array}{l}\text { Staphylococcus aureus } \\
\text { resistente a meticilina/ } \\
\text { Pseudomonas aeruginosa }\end{array}$ & 33 & 20,2 & 64,7 \\
\hline
\end{tabular}

CVF\%: Capacidade vital forçada ;VEF\%: Volume expiratório forçado cronometrado de primeiro segundo (VEF1) ;CVF/VEF\%: Razão entre volume expiratório forçado (cronometrado) e capacidade vital forçada; IMC: índice de massa corpórea

Tabela 2. Análise comparativa dos dispositivos Shaker $\AA^{\circledR}$ e Quake ${ }^{\circledR}$ em relação aos parâmetros cardiorrespiratórios, peso seco e úmido da secreção.

\begin{tabular}{|c|c|c|c|c|}
\hline \multirow[b]{2}{*}{ Variáveis } & & Shaker ${ }^{\circledR}$ & Quake® & \multirow[b]{2}{*}{ Valor $p$} \\
\hline & & $\begin{array}{l}\text { Mediana/ intervalo } \\
\text { interquartil }\end{array}$ & $\begin{array}{l}\text { Mediana/ intervalo } \\
\text { interquartil }\end{array}$ & \\
\hline \multirow{3}{*}{$\begin{array}{l}\text { Peso úmido } \\
\text { gramas }\end{array}$} & Pré & $1,35(1,9)$ & $1,8(2,26)$ & $\mathrm{p}^{\mathrm{x}}: 0,26$ \\
\hline & Pós & $3,91(5,13)$ & $4,3(6,4)$ & $\mathrm{p}^{\mathrm{x}}: 0,48$ \\
\hline & & $p^{x}: 0,017$ & $p^{x}: 0,036$ & \\
\hline \multirow{3}{*}{$\begin{array}{l}\text { Peso Seco } \\
\text { gramas }\end{array}$} & Pré & $0,35(0,31)$ & $0,6(0,55)$ & $p^{x}: 0,26$ \\
\hline & Pós & $0,56(0,32)$ & $0,6(1,1)$ & $\mathrm{p}^{\mathrm{x}}: 0,57$ \\
\hline & & $p^{x}: 0,16$ & $p^{x}: 0,4$ & \\
\hline \multirow{3}{*}{$\begin{array}{l}\text { Frequência Cardíaca } \\
\text { bpm }\end{array}$} & Pré & $108(11,2)$ & $104(9,7)$ & $p^{x}: 1,0$ \\
\hline & Pós & $111(24)$ & $111(44,5)$ & $p^{x}: 0,89$ \\
\hline & & $p^{x}: 0,20$ & $p^{x}: 0,3$ & \\
\hline \multirow{3}{*}{$\begin{array}{l}\text { Frequência Respiratória } \\
\text { ipm }\end{array}$} & Pré & $30(10,7)$ & $27(16)$ & $p^{x}: 1,0$ \\
\hline & Pós & $32(19,0)$ & $33(26)$ & $p^{x}: 0,14$ \\
\hline & & $p^{x}: 0,024$ & $p^{x}: 0,012$ & \\
\hline \multirow{3}{*}{$\begin{array}{l}\text { Percepção subjetiva de } \\
\text { esforço }\end{array}$} & Pré & $0(0)$ & $0(0,88)$ & $p^{x}: 1,0$ \\
\hline & Pós & $0,75(3,5)$ & $1(2,5)$ & $p^{x}: 0,91$ \\
\hline & & $p^{\times}: 0,06$ & $p^{x}: 0,042$ & \\
\hline \multirow{3}{*}{$\begin{array}{c}\mathrm{SpO} 2 \\
\%\end{array}$} & Pré & $95(8)$ & $97(9,75)$ & $p^{x}: 0,78$ \\
\hline & Pós & $95(8)$ & $96(8,7)$ & $\mathrm{p}^{\mathrm{x}}: 0,49$ \\
\hline & & $p^{x}: 0,3$ & $p^{x}: 0,7$ & \\
\hline
\end{tabular}

px: Wilcoxon; bpm: batimentos por minuto, ipm: incursões por minuto. 

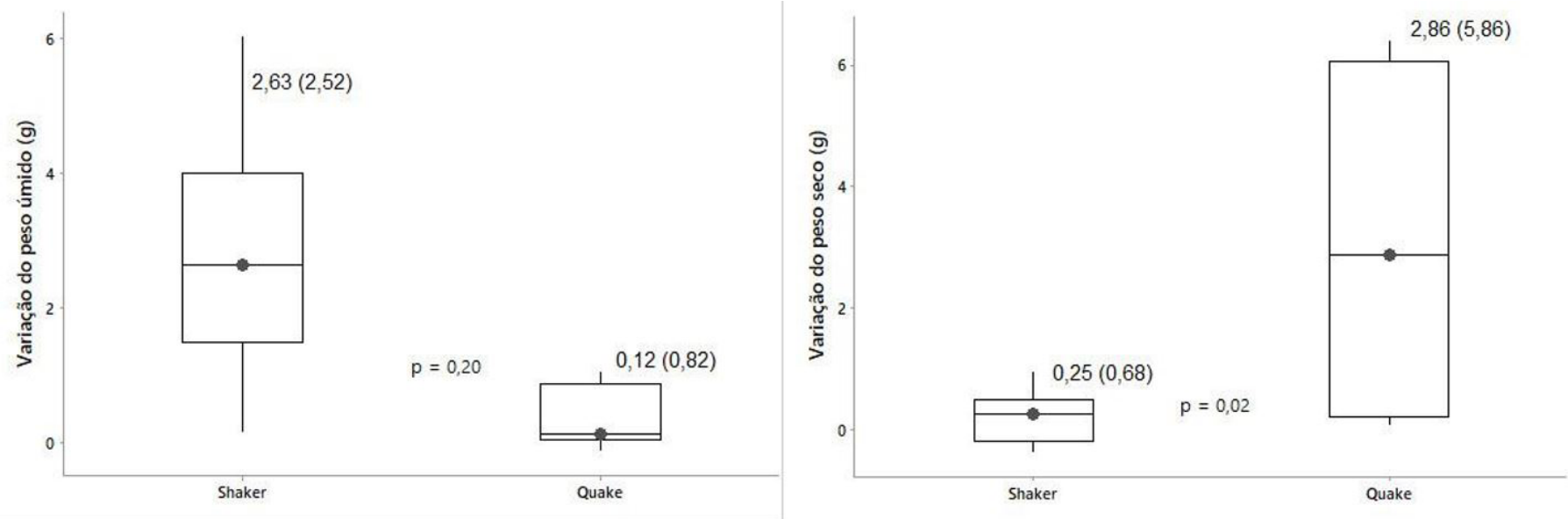

Gráfico 1. Diferença de peso seco e úmido com uso dos dispositivos.

representado pelo boxplot. O dispositivo Quake ${ }^{\circledR}$, foi mais eficiente na remoção das secreções pulmonares.

Quando avaliado o efeito de cada intervenção sobre o peso úmido e Fr, foi observado um aumento das variáveis após a realização dos dispositivos. Os participantes relataram um aumento na percepção do esforço apenas após a realização do Quake ${ }^{\circledR}$. Não foram observadas diferenças estatísticas nas variáveis peso seco, $\mathrm{Fc}$ e $\mathrm{SpO}_{2}$ após a realização dos dois dispositivos.

$\mathrm{Na}$ avaliação da preferência entre os dispositivos, sete pacientes relataram preferir o Quake ${ }^{\circledR}$ e um paciente o Shaker ${ }^{\circledR}$

\section{DISCUSSÃO}

Os resultados deste estudo demonstram que os dispositivos Shaker ${ }^{\circledR}$ e Quake ${ }^{\circledR}$ apresentam efeitos semelhantes nos parâmetros cardiorrespiratórios avaliados e são equivalentes com relação à remoção das secreções brônquicas.

Na comparação do delta de variação do peso seco entre Shaker ${ }^{\circledR}$ e Quake ${ }^{\circledR}$, houve uma diferença significativa entre os dispositivos. O delta de variação do Quake ${ }^{\circledR}$ foi significativamente maior. Não é de nosso conhecimento estudos que avaliaram e compararam essas variáveis em crianças e adolescentes com fibrose cística.

A oscilação oral de alta frequência combina os benefícios da pressão positiva na expiração com as vibrações das vias aéreas ${ }^{5}$. Durante sua utilização promovem uma expiração contra resistência, criando oscilações nas vias aéreas o que impede o seu colapso, além de promover alterações nas propriedades viscoelásticas do muco, tornando fácil sua mobilização e eliminação de secreção ${ }^{10}$.

O Shaker ${ }^{\circledR}$ é um protótipo nacional do Flutter VRP1 ${ }^{\circledR}$, de baixo custo, desenvolvido com objetivo de favorecer a remoção das secreções pulmonares ${ }^{11}$.

O Quake ${ }^{\circledR}$ é um aparelho portátil, desenvolvido em 2006 (Thayer Medical, Tocson, USA) com objetivo de promover a remoção das secreções brônquicas. Constituído de material plástico em formato de cachimbo, com uma manivela giratória, operado manualmente pelo paciente, que cria as oscilações responsáveis pela geração da pressão positiva expiratória5-9

Okeson e McGowen 12, 2007 realizaram um estudo comparando a força de pressão de pulso e frequência de vibração em vários ângulos, gerada pelos dispositivos Acapella, Flutter VRP1 $₫$ e Quake ${ }^{\circledR}$ em um simulador de baixo volume pulmonar. Os autores encontraram maiores pressões de pulso com o uso do Quake ${ }^{\circledR}$, que indica maior vibração nas vias aéreas e facilita a eliminação de secreção.

Um estudo com metodologia semelhante foi realizado por Santos et al. ${ }^{14}$ que comparou o desempenho mecânico do Shaker ${ }^{\circledR}$, Flutter VRP1 ${ }^{\circledR}$ e Acapella em diferentes inclinações e fluxos. Ao contrário dos achados anteriores, todos os dispositivos tiveram efeitos semelhantes, produzindo pressões e oscilações consideradas eficazes na desobstrução brônquica. No entanto, os estudos foram feitos em modelos experimentais sem intervenções clínicas ${ }^{14}$.

Em um estudo recente, Suzan et al. ${ }^{6}$ compararam os efeito do Flutter VRP1 ${ }^{\circledR}$ e Shaker ${ }^{\circledR}$, na quantidade de secreção pulmonar expectorada e nos parâmetros cardiorrespiratórios em pacientes com fibrose cística. Foram analisados os seguintes aspectos: $\mathrm{Fc}, \mathrm{Fr}, \mathrm{SpO}_{2^{\prime}}$ Escore de Schwachman e preferência quanto a utilização. Os resultados demonstraram que não houve diferenças significativas entre os dispositivos nas variáveis avaliadas e atestam que os dispositivos possuem comportamento semelhante na remoção das secreções pulmonares ${ }^{6}$.

Coelho et al. ${ }^{9}$ estudaram voluntários que utilizaram o dispositivo Quake ${ }^{\circledR}$, mas as variáveis estudadas foram a modulação no padrão respiratório e o impacto nas pressões geradas sem a avaliação sobre a remoção das secreções. Esses autores observaram que a pressão positiva expiratória máxima gerada pelo Quake ${ }^{\circledR}$ varia de acordo com o número de rotações por minuto. $\mathrm{Na}$ sequência de 20 incursões por minuto, as pressões 
geradas foram mais homogêneas e ocasionaram maior cansaço dos voluntários ${ }^{9}$.

No presente estudo, os pacientes foram orientados a manter uma rotação da manivela em torno de 20 incursões por minutos, que contribuiu para o aumento das pressões geradas pelo dispositivo Quake ${ }^{\circledR}$ e como consequência foi observado um aumento da $\mathrm{Fr}$ e da percepção do esforço. Outro achado importante foi a preferência dos pacientes na realização do Quake®. Esse tipo de avaliação em estudos clínicos tem sido utilizada como desfecho positivo ${ }^{15}$. A preferência deve ser considerada, pois é um importante aspecto na adesão ao tratamento ${ }^{16}$. No protocolo de tratamento do centro de referência em que este estudo foi realizado ocorre a disponibilização do Shaker ${ }^{\circledR}$ para os usuários. Portanto, os participantes avaliados já utilizavam em sua rotina este dispositivo. $\mathrm{O}$ uso de um recurso diferente pode despertar o interesse para sua utilização, o que pode justificar a preferência dos participantes deste estudo pelo Quake®.

O peso úmido é o volume de secreção pulmonar expectorado que é utilizado para quantificar o efeito de técnicas de fisioterapia respiratória na remoção das secreções pulmonares. Entretanto, é uma medida que pode sofrer alterações decorrentes da quantidade de saliva eliminada. Por isso, neste estudo medidas do peso seco e úmido foram realizadas ${ }^{13}$.

$\mathrm{Na}$ avaliação do peso úmido foi observado que os dispositivos promoveram a remoção da secreção brônquica de forma semelhante e foram eficientes para esta finalidade. Não é de nosso conhecimento estudos que avaliam os desfechos de parâmetros cardiorrespiratórios e remoção de secreção das vias aéreas com a utilização do Quake ${ }^{\circ}$, portanto, o presente trabalho é pioneiro em atestar a superioridade deste dispositivo na remoção das secreções pulmonares.

\section{CONCLUSÃO}

O dispositivo Quake ${ }^{\circledR}$ apresentou superioridade na remoção de secreções das vias aéreas em pacientes com fibrose cística. Os voluntários relataram preferência na sua utilização. Na avaliação dos parâmetros cardiorrespiratórios não foram encontradas diferenças estatisticamente significativas entre os dois aparelhos de pressão positiva oscilatórias avaliados.

\section{FONTE DE FINANCIAMENTO}

Estudo financiado pelos pesquisadores e apoio institucional da Fundação Hospitalar do Estado de Minas Gerais

\section{CONFLITO DE INTERESSES}

Aquino ES recebeu os dispositivos Quake como doação pela empresa Thayer Medical. A empresa não teve qualquer envolvimento na análise dos dados ou na redação deste artigo. Carmo FDMR, Coelho CC e Januário RCV nenhum conflito de interesse a declarar.

\section{REFERÊNCIAS}

1. Castellani C, Assael M. Cystic fibrosis: a clinical view. Cell Mol Life Sci. 2017 jan;74(1):129-40. http://dx.doi.org/10.1007/ s00018-016-2393-9. PMid:27709245.

2. Palma A, Kotsias B, Marino G. Funciones de los canales iónicos cftr y enac en la fibrosis quística. Medicina (B. Aires). 2014 abr;74(2):133-9.

3. Ratjen FA. Cystic fibrosis: pathogenesis and future treatment strategies. Respir Care. 2009 maio;54(5):595-605. http:// dx.doi.org/10.4187/aarc0427. PMid:19393104.

4. Rosa F, Dias F, Nobre L, Morais H. Cystic fibrosis: a clinical and nutritional approach. Rev. Nutr. 2008 nov-dez;21(6):725-737

5. Myers TR. Positive expiratory pressure and oscillatory positive expiratory pressure therapies. Respir Care. 2007 out;52(10):1308-26, discussion 1327. PMid:17894901.

6. Suzan A, Galvão F, Ribeiro A, Ribeiro J, Ribeiro M, Assumpção $M$, et al. Oscilação oral de alta frequência e fibrose cística: comparação entre Instrumentais. Conscientiae Saúde. 2015;14(2):283-90. http://dx.doi.org/10.5585/conssaude. v14n2.5418.

7. Bradley JM, Kent L, O'neill B, Nevill A, Boyle L, Elborn S. Cardiorespiratory measurements during field tests in $\mathrm{CF}$ : use of an ambulatory monitoring system. Pediatr Pulmonol. 2011;46(3):253-60. http://dx.doi.org/10.1002/ppul.21360. PMid:20967834.

8. Duarte PECR, Silva VL, Silva DAL. Análise do funcionamento do Shaker® à diversos níveis de fluxo. Pulmäo RJ. 2014;16(24):70-5.

9. Coelho CC, Diniz A, Carvalho LMM, Reis RM, Mourão FAG, et al. Avaliação da pressão positiva expiratória final utilizando o aparelho fisioterápico Quake. Fisioterapia e Pesquisa. 2009;16(4):352-6.

10. Morrison L, Agnew J. Oscillating devices for airway clearance in people with cystic fibrosis. Cochrane Database Syst Rev. 2017;7(1):CD006842. http://dx.doi.org/10.1002/14651858. CD006842.pub2.

11. Suzan AB. Comparação entre a eficácia de dois aparelhos utilizados na fisioterapia respiratória em pacientes com fibrose cística (dissertação). Campinas: Faculdade de Ciências Médicas, Universidade Estadual de Campinas; 2006

12. Okeson C, McGowen P. The percussive characteristics of the Acapella, Flutter and Quake During Low-Volume Tidal Breathing. Chicago: American College of Chest Physicians; 2007

13. Prasad S, Main E. Finding evidence to support airway clearance techniques in cystic fibrosis. Disabil Rehabil. 1998 jun-jul;20(6-7):235-46. http://dx.doi. org/10.3109/09638289809166734. PMid:9637932.

14. Santos AP, Guimarães RC, Carvalho EM, Gastaldi AC. Mechanical behaviors of Flutter VRP1, Shaker, and Acapella devices. Respir Care. 2013 fev;58(2):298-304. http://dx.doi. org/10.4187/respcare.01685.

15. Mcllwaine M, Button B, Dwan K. Positive expiratory pressure physiotherapy for airway clearance in people with cystic fibrosis. Cochrane Database Syst Rev. 2015 jun 17;(6):CD003147. http://dx.doi.org/ 10.1002/14651858. CD003147.pub4.

16. Flume PA, Robinson KA, O'Sullivan BP, Finder JD, Vender $R L$, Willey-Courand $D-B$, et al. Cystic fibrosis pulmonary guidelines: aieway clearence therapies. Respir Care, 2009 abr;54(4):522-37. PMID: 19327189. 